University of South Carolina

Scholar Commons

8-22-2013

\title{
A Multi-Physics Model for Solid Oxide Iron-Air Redox Flow Battery: Simulation of Discharge Behavior at High Current Density
}

\author{
Meng Guo \\ University of South Carolina - Columbia, guom@mailbox.sc.edu \\ Xuan Zhao \\ Ralph E. White \\ University of South Carolina - Columbia, white@cec.sc.edu \\ Kevin Huang \\ University of South Carolina - Columbia, huang46@cec.sc.edu
}

Follow this and additional works at: https://scholarcommons.sc.edu/eche_facpub

Part of the Chemical Engineering Commons

\footnotetext{
Publication Info

Published in Journal of The Electrochemical Society, Volume 160, Issue 11, 2013, pages A2085-A2092. (C) Journal of The Electrochemical Society (2013), The Electrochemcial Society.

(C) The Electrochemical Society, Inc. 2013. All rights reserved. Except as provided under U.S. copyright law, this work may not be reproduced, resold, distributed, or modified without the express permission of The Electrochemical Society (ECS). The archival version of this work was published in Journal of The Electrochemical Society.

Publisher's Version: http://dx.doi.org/10.1149/2.062311jes

- Guo, M., Zhao, X., White, R. E., \& Huang, K. (August 22, 2013). A Multi-Physics Model for Solid Oxide IronAir Redox Flow Battery: Simulation of Discharge Behavior at High Current Density. Journal of The Electrochemical Society, 160 (11), A2085 - A2092. http://dx.doi.org/10.1149/2.062311jes
}

This Article is brought to you by the Chemical Engineering, Department of at Scholar Commons. It has been accepted for inclusion in Faculty Publications by an authorized administrator of Scholar Commons. For more information, please contact digres@mailbox.sc.edu. 


\title{
A Multi-Physics Model for Solid Oxide Iron-Air Redox Flow Battery: Simulation of Discharge Behavior at High Current Density
}

\author{
Meng Guo, ${ }^{\mathrm{a}}$ Xuan Zhao, ${ }^{\mathrm{b}, *}$ Ralph E. White, ${ }^{\mathrm{a}, * *, \mathrm{z}}$ and Kevin Huang ${ }^{\mathrm{b}, * * *}$ \\ ${ }^{a}$ Department of Chemical Engineering, University of South Carolina, Columbia, South Carolina 29208, USA \\ ${ }^{b}$ Department of Mechanical Engineering, University of South Carolina, Columbia, South Carolina 29208, USA
}

\begin{abstract}
A rigorous physics-based mathematical model for a solid oxide iron-air redox flow battery system is presented in this paper. The modeled flow battery system combines a $\mathrm{Fe}-\mathrm{FeO}$ redox couple as the energy storage unit and a regenerative solid oxide fuel cell as the electrical functioning unit in a 2D axial symmetric geometry. This model was developed from fundamental theories of reaction engineering in which basic transport phenomena and chemical/electrochemical kinetics are included. The model shows good agreement with the experimental data. Simulation results for the chemical, electrochemical and transport behavior of the battery are discussed.
\end{abstract}

(C) 2013 The Electrochemical Society. [DOI: 10.1149/2.062311jes] All rights reserved.

Manuscript submitted July 16, 2013; revised manuscript received August 22, 2013. Published October 2, 2013.

Rechargeable batteries represent a class of advanced electrical energy storage (EES) mechanisms that can be hybridized with other EES devices in a smart grid. Among them, metal-air batteries have attracted much attention in recent years due to their intrinsically high energy density and needless storage of air reactant at the cathode. ${ }^{1-4}$ Recently, a distinct group of solid oxide redox flow batteries targeted at stationary energy storage was developed based on the technologies of reversible solid oxide fuel cell (RSOFC) and $\mathrm{H}_{2}$ chemical looping. ${ }^{5-17}$ The nature of this type of metal-air battery is the use of solid $\mathrm{O}^{2-}$ conducting electrolyte. The uniqueness of this new battery is manifested by the fact that the electrical energy conveyed by RSOFC can be reversibly converted into chemical energy by a physically separated metal-metal oxide $\left(\mathrm{Me}-\mathrm{MeO}_{\mathrm{x}}\right)$ redox couple. Such an in-situ conversion can lead to high efficiency and easy system integration. In addition, separation of the energy storage function from the electrodes tolerates a large degree of physical volume change occurring in redox couple during electrical cycles without impacting the battery's structural integrity.

As experimental studies on the characteristics of the new battery are currently being carried out in our group, it becomes increasingly clear that a fundamental understanding of the complex physical, chemical, mechanical, and electrochemical phenomena involved in the system would greatly benefit the development of the battery. Physics-based mathematical modeling would be an ideal tool to meet this demand. So far, very little modeling work on this type of battery has been reported. In 2012, Ohmoti et al ${ }^{18}$ proposed a 1D Fick's diffusion model with the simplest configuration to study the battery's characteristics. However, that model has at least two major limitations. First, the geometries for most practical designs should be beyond 1D. Second, the transport phenomena in the concentrated gas phase are far more complicated than the Fick's diffusion. Therefore, the major objective of this work is to develop a more rigorous multi-physics model than the Ohmoti's 1D model, which would encompass most of the transport and kinetic processes involved in the solid oxide redox flow batteries. The theoretical basis for the model development is the fundamentals in the reaction engineering and chemical reactor design. ${ }^{19}$ The outcome of the constructed model is also compared with the measured experimental results.

\section{Mathematical Model}

Working principles of the flow battery system.- The details for the working principles and chemical/electrochemical mechanisms of the solid oxide redox flow battery can be found in ref. 5-18 Although the operating temperature, geometries and configurations for

\footnotetext{
*Electrochemical Society Student Member

***Electrochemical Society Fellow.

*** Electrochemical Society Active Member.

${ }^{\text {z} E-m a i l: ~ w h i t e @ c e c . s c . e d u ~}$
}

the flow battery systems reported in the literature vary to some degree, our multi-physics model presented here is developed for a close-loop tubular battery reactor working at $800^{\circ} \mathrm{C}$. According to the schematic illustrated in Figure 1, this flow battery system includes a cylindrical tubular reactor that carries out the battery's functionality and a gas manifold that includes a "T"-shape tube and a returning tubing that allow the gas mixture leaving and circulating back to the tubular reactor. The tubular reactor can be regarded as the combination of a RSOFC and a redox cycle unit (RCU). The RSOFC has a planar disk configuration and is sealed at the top of the tubular reactor with the anode (negative electrode) facing the interior space of the tubular reactor. Right below the RSOFC is the RCU, a packed-bed containing porous $\mathrm{Fe}$-based redox-couple pellets. The circulating gas is a mixture of $\mathrm{H}_{2}$ and $\mathrm{H}_{2} \mathrm{O}$, and the direction of close-loop flow is shown by the green arrows in Figure 1. At the anode of RSOFC, the following electrochemical reaction occurs between the hydrogen and the steam:

$$
\mathrm{H}_{2}+\mathrm{O}^{2-} \underset{\text { charge }}{\stackrel{\text { discharge }}{\rightleftharpoons}} \mathrm{H}_{2} \mathrm{O}+2 e^{-}
$$

The cathode (positive electrode) of the RSOFC is an air electrode facing the outside of the tubular reactor, and the following electrochemical reaction occurs at the cathode:

$$
\frac{1}{2} \mathrm{O}_{2}+2 e^{-} \underset{\text { charge }}{\stackrel{\text { discharge }}{\rightleftharpoons}} O^{2-}
$$

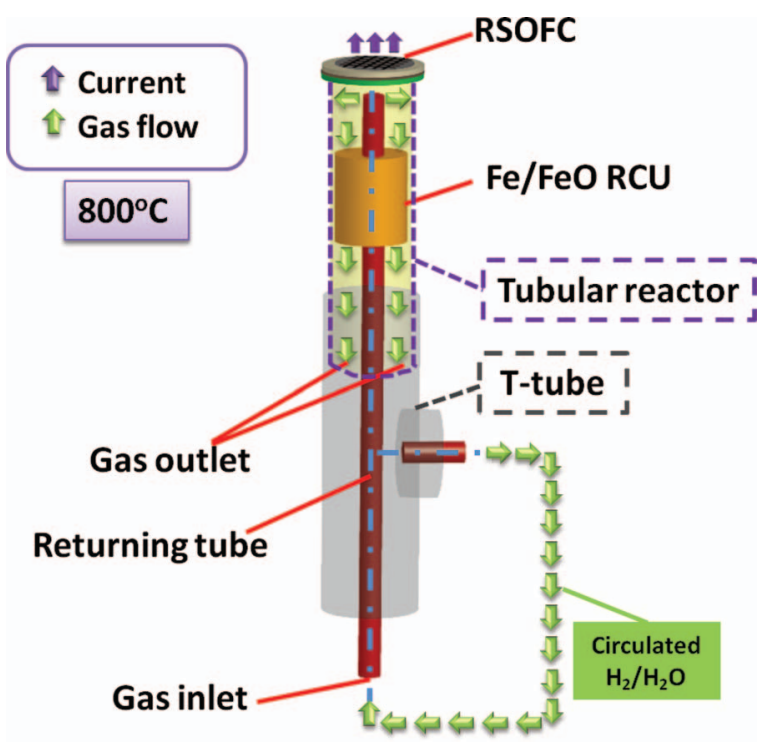

Figure 1. Configuration and operating principle for the solid oxide redox flow battery. 


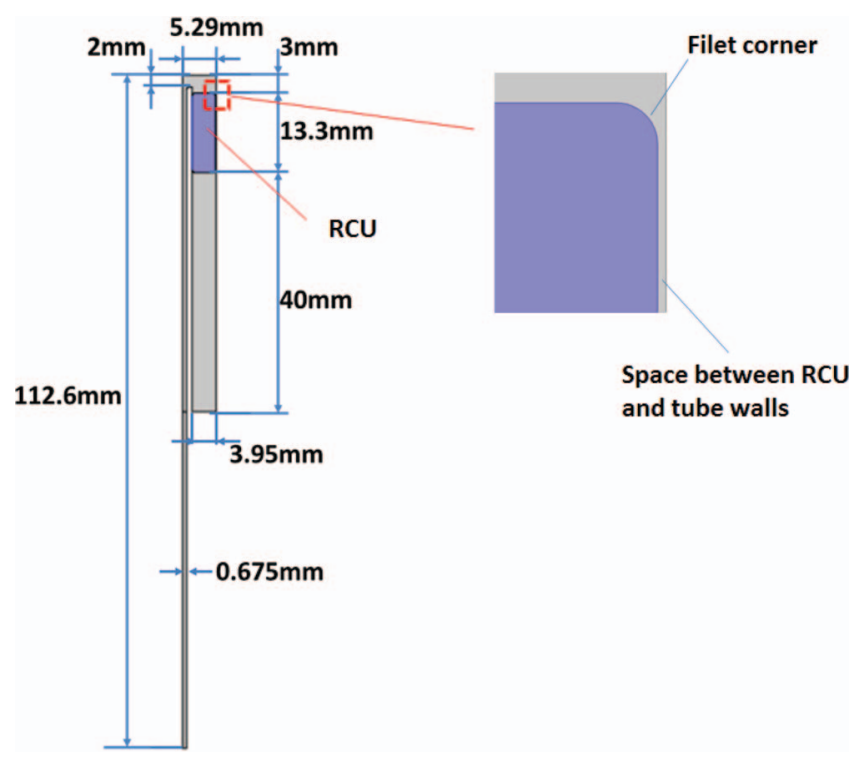

Figure 2. Cross-section of the modeling domains for the flow battery in a $2 \mathrm{D}$ plane.

In the RCU, the following reversible homogeneous reaction occurs between the solid and gas phases:

$$
\mathrm{H}_{2} \mathrm{O}+\mathrm{Fe} \underset{\text { charge }}{\stackrel{\text { discharge }}{\rightleftharpoons}} \mathrm{H}_{2}+\mathrm{FeO}
$$

The gas leaves the tubular reactor from the bottom (the gas outlet) and is collected in the T-tube; then the gas is recirculated back to the tubular reactor through the returning tube.

As shown above, the gaseous species are consumed at the RSOFC and regenerated in the RCU, and then re-supplied to the battery system. Therefore, the capacity of this flow battery is theoretically limited only by the total loading of the redox couple $\mathrm{Fe} / \mathrm{FeO}$.
Basic assumptions and the model simplification.- - In this work, a mathematical model was developed for the system using COMSOL 4.3 multi-physics software. The whole system is regarded as isothermal, and therefore, the thermal diffusion of the gaseous species and the heat transfer are neglected. The transport phenomena in the model include the computational fluid dynamics (CFD) and the convective mass transfer of concentrated species, ${ }^{20,21}$ and the gas flow is assumed to be laminar. For a complete model, the T-tube has to be treated as a 3D fluid domain due to the asymmetric geometry; however, this would make this model computationally too expensive. To avoid the large computation loads and make the simulations more efficient, we simplified the model by regarding the T-tube as a continuous-stirred tank (CST). The gas phase in the CST is well-stirred under a constant pressure; therefore, the species in the gas phase can be assumed to have uniformly-distributed concentration profiles throughout the volume of CST. The mass balance for the CST is thus reduced to lumped submodels. Therefore, the mass and momentum distributions treated by the simplified model include only the tubular reactor and the returning tube.

Computational domains and model settings. - As the tubular reactor and the returning tube are co-axial cylinders, the computational domains of this model can be built on a two-dimensional axial-symmetric plane. The schematic for the in-plane cross-section of the modeling domains is presented in Figure 2 and the full 3D geometry for the tubular reactor and the returning tube can be obtained by revolving this cross-section for $360^{\circ}$ around the symmetric axis. In order to improve the gas recirculation in the tubular reactor, corner fillets are applied for RCU domain and small space is also left between the exterior vertical boundary of the RCU and the wall of the tubular reactor.

Figure 3a shows the settings of physical sub-models for different domains and boundaries. Regions outside the RCU contain only the gas phase and are set as free-flow domains. The RCU domain, which includes both solid and gas phases, is regarded as a porous media in which the transport properties are scaled by the porosity and the tortuosity. As the regenerative homogeneous redox reactions occur in the RCU domain, volumetric mass sources are coupled to the transport equations. Due to the small thickness, the RSOFC is

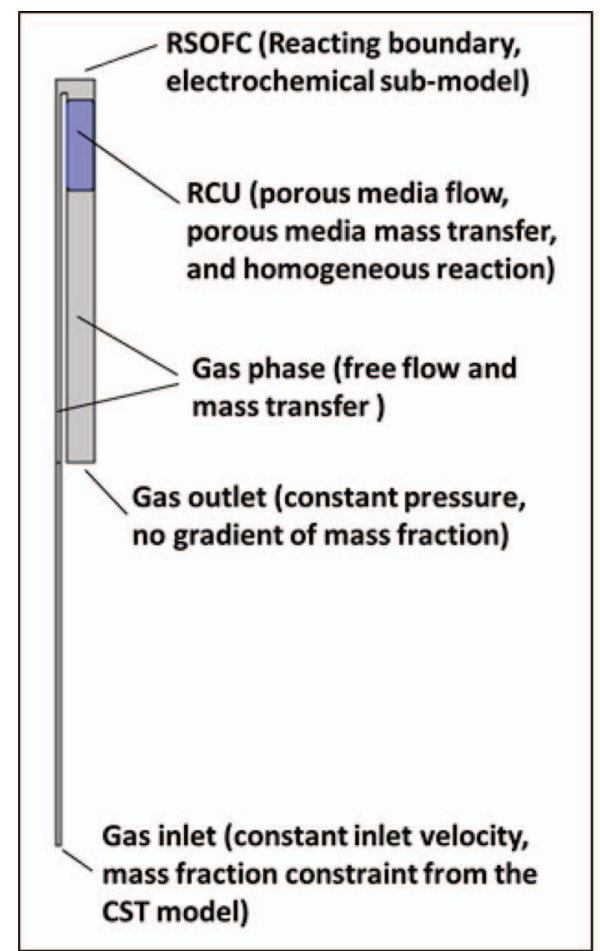

(a) Domain sub-model settings

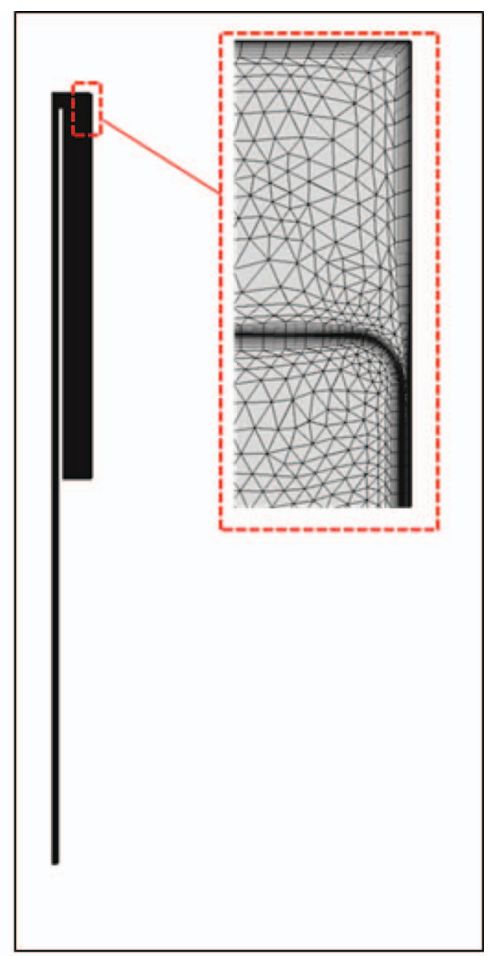

(b) Meshing mode
Figure 3. Basic model settings: (a) physical submodels for computational domains and boundaries, (b) the meshing patterns. 
regarded as a reacting boundary where the mass flux of the gas phase species is proportional to the anode electrochemical current density. The voltage-current characteristic of the RSOFC is governed by the electrochemical sub-models. The gas circulation through the "T"-tube is simplified into a lumped sub-model that formulates the gas phase compositions in the inflow and outflow. The walls of the tubular reactor and the returning tube are set as no-slip and insulated boundaries, and the central axis is set as a symmetric boundary. The meshing of the model domains are presented in Figure 3b; to ensure sufficient computation accuracy, extremely fine meshing is used in this work and the discretized domains include totally 24,611 domain elements and 1,635 boundary elements. Quadrilateral mesh was selected for the near-wall boundary layers and triangular mesh was applied for all the other regions. The derivations of the model equations are presented in the following subsections.

The mass and momentum transports in the free-flow phase.-In the free-flow phase, the multi-component mass transfer for concentrated species is governed by the Maxwell-Stefan diffusion and convection, and the governing equation is as follow:

$$
\rho \frac{\partial \omega_{i}}{\partial t}+\nabla \cdot \underline{\mathbf{j}}_{i}+\rho(\underline{\mathbf{u}} \cdot \nabla) \omega_{i}=0 \quad(i=1,2)
$$

where $\omega_{i}$ is the mass fraction of species $i, \rho$ is the density of gas phase, $\mathbf{j}$ is the diffusive mass flux vector for species $i, \underline{\mathbf{u}}$ is the velocity vector of the fluid flow, and the subscript $i=1$ stands for hydrogen and $i=2$ stands for steam. The expression for diffusive mass flux vector, $\underline{\mathbf{j}}_{i}$ (in the unit of $\mathrm{kg} \cdot \mathrm{m}^{-2} \cdot \mathrm{s}^{-1}$ ), is as follow:

$$
\underline{\mathbf{j}}_{i}=-\rho \omega_{i} \sum_{k} \widetilde{D}_{i, k} \underline{\mathbf{d}}_{k} \quad(i=1,2 \text { and } k=1,2)
$$

Where $\tilde{D}_{i, k}$ are the multi-component Fick's diffusivities, $\underline{\mathbf{d}}_{k}$ (in the unit of $1 / \mathrm{m}$ ) is the diffusive driving force acting on species $k$, and subscript $k$ is a dummy index for species. According to ref. 20, the values of $\tilde{D}_{i, k}$ can be calculated from the multi-component MaxwellStefan diffusivities, $D_{i, j}$ (where $i \neq j$ and $D_{i, j}=D_{j, i}$ ); therefore for this binary system, only the value of $D_{1,2}$ is needed to implement the model. In this work, the hydrogen/steam mixture is regarded as ideal gas and the diffusive driving forces are expressed as:

$$
\underline{\mathbf{d}}_{k}=\nabla x_{k}+\frac{1}{p_{A}}\left[\left(x_{k}-\omega_{k}\right) \nabla p_{A}\right]
$$

where $x_{k}$ is the mole fraction of species $k$ and $p_{A}$ is the absolute pressure. The mole fraction $x_{k}$ can be calculated as:

$$
\begin{gathered}
x_{k}=\frac{\omega_{k}}{M_{k}} M_{n} \\
M_{n}=\left(\sum_{k} \frac{\omega_{k}}{M_{k}}\right)^{-1}
\end{gathered}
$$

where $M_{k}$ is the molar mass for species $k$ and $M_{n}$ is the mean Molar mass for the gas mixture. The total mass flux of species $i, \underline{\mathbf{N}}_{i}$, is the combination of the diffusive flux and the convective flux:

$$
\underline{\mathbf{N}}_{i}=\underline{\mathbf{j}}_{i}+\rho \underline{\mathbf{u}} \omega_{i} \quad(i=1,2)
$$

The fluid dynamics in the free-flow phase ${ }^{22}$ are described by the Navier-Stokes equations:

$$
\rho \frac{\partial \underline{\mathbf{u}}}{\partial t}+\rho(\underline{\mathbf{u}} \cdot \nabla) \underline{\mathbf{u}}=\nabla \cdot\left\{-p \underline{\underline{\mathbf{I}}}+\mu\left[\nabla \underline{\mathbf{u}}+(\nabla \underline{\mathbf{u}})^{\tau}\right]-\frac{2}{3} \mu(\nabla \cdot \underline{\mathbf{u}}) \underline{\underline{\mathbf{I}}}\right\}
$$

and the continuity equation:

$$
\frac{\partial \rho}{\partial t}+\nabla \cdot(\rho \underline{\mathbf{u}})=0
$$

where $\mu$ is the viscosity of the gas phase, $\underline{\underline{I}}$ is the identity matrix, and $p$ is the modified pressure which is defined as:

$$
p=p_{A}-p_{\text {ref }}
$$

and in this work, the reference pressure, $p_{\text {ref }}$, is set at $1 \mathrm{~atm}$.

The mass and momentum transports in the RCU domain.- In the RCU domain, the gas phase is regarded as reacting-flow in a porous media, and the mass transfer governing equations in the gas phase are as follow:

$$
\begin{gathered}
\varepsilon_{p} \rho \frac{\partial \omega_{i}}{\partial t}+\nabla \cdot \underline{\mathbf{j}}_{i}+\rho(\underline{\mathbf{u}} \cdot \nabla) \omega_{i}=R_{i} \quad(i=1,2) \\
\underline{\mathbf{j}}_{i}=-\rho \omega_{i} \sum_{k} \widetilde{D}_{i, k}^{\text {eff }} \underline{\mathbf{d}}_{k} \quad(i=1,2 \text { and } k=1,2)
\end{gathered}
$$

where $\varepsilon_{p}$ is the porosity of RCU and $\tilde{D}_{i, k}^{e f f}$ are the effective multicomponent Fick's diffusivities, and $R_{i}$ is the volumetric mass source of species $i$. The expressions for effective diffusivities are as follow:

$$
\widetilde{D}_{i, k}^{\text {eff }}=D_{i, k} \varepsilon_{p}^{3 / 2} \quad(i=1,2 \text { and } k=1,2)
$$

According to the reaction eq. 3 , the rate for this multi-phase chemical reaction depends on the concentrations of reactants in both the gas phase (hydrogen and steam) and the solid phase ( $\mathrm{Fe}$ and $\mathrm{FeO}$ ). Let $\xi_{\mathrm{Fe}}$ stand for the conversion of $\mathrm{Fe}$ to $\mathrm{FeO}$, it can be easily found that the molar fractions of $\mathrm{Fe}$ and $\mathrm{FeO}$ are respectively proportional to $1-\xi_{\mathrm{Fe}}$ and $\xi_{F e}$. Assuming that the reaction orders equal the stoichiometric coefficients in eq. 3 , the volumetric molar reaction rates for hydrogen and steam, $r_{1}$ and $r_{2}$, can thus be expressed as:

$$
r_{1}=k\left(1-\xi_{F e}\right) c_{2}-k^{-1} \xi_{F e} c_{1}
$$

$$
r_{2}=-r_{1}
$$

where $c_{1}$ and $c_{2}$ are the molar concentrations of hydrogen and steam, and $k$ and $k^{-1}$ (in units of 1/s) are respectively the rate constants for the forward and reverse reactions. For species $i$, the volumetric mass source terms, $R_{i}$, is the molar reaction rate scaled by the Molar mass, $M_{i}$ :

$$
R_{i}=r_{i} M_{i} \quad(i=1,2)
$$

According to the ideal gas properties, the molar concentrations of species $i$ can be calculated from the mole fraction:

$$
c_{i}=\frac{x_{i} p_{A}}{R T} \quad(i=1,2)
$$

where $T$ is temperature $(\mathrm{K})$ and $R$ is the universal gas constant. The governing equation for the mass balance in the solid phase is as follow:

$$
c_{F e} \frac{\partial \xi_{F e}}{\partial t}=r_{1}
$$

where $c_{F e}$ is the total amount of $\mathrm{Fe}$ and $\mathrm{FeO}$ per unit bulk volume of RCU. Therefore, $c_{F e}$ is expressed as:

$$
c_{F e}=\frac{n_{F e}+n_{F e O}}{V_{\mathrm{RCU}}}
$$

where $n_{\mathrm{Fe}}$ and $n_{\mathrm{FeO}}$ (in the unit of mole) are the amounts of Fe and $\mathrm{FeO}$, and $V_{\mathrm{RCU}}$ is the bulk volume of RCU. As the conversion between $\mathrm{Fe}$ and $\mathrm{FeO}$ is equal-molar, the value for $n_{\mathrm{Fe}}+n_{\mathrm{FeO}}$ remains constant and is equal to the total number of mole of $\mathrm{Fe}$ and $\mathrm{FeO}$ in the initial RCU loading. As $\mathrm{Fe}$ and $\mathrm{FeO}$ have different molar volume values, the porosity of RCU, $\varepsilon_{p}$, may change with the progress of reaction 3 , and the correlation between $\varepsilon_{p}$ and $\xi_{F e}$ is derived as follow:

$$
\varepsilon_{p}=\varepsilon_{p, 0}+c_{F e}\left(\frac{M_{F e}}{\rho_{F e}}-\frac{M_{F e O}}{\rho_{F e O}}\right)\left(\xi_{F e}-\xi_{F e, 0}\right)
$$

where $\varepsilon_{p, 0}$ is the initial porosity of $\mathrm{RCU}, \xi_{F e, 0}$ is the initial conversion of $\mathrm{Fe}$ to $\mathrm{FeO}, M_{\mathrm{Fe}}$ and $M_{\mathrm{FeO}}$ are the Molar mass of $\mathrm{Fe}$ and $\mathrm{FeO}$, and $\rho_{\mathrm{Fe}}$ and $\rho_{\mathrm{Fe} O}$ are the densities of $\mathrm{Fe}$ and $\mathrm{FeO}$, respectively. 
The fluid dynamics of the gas phase in the RCU domain are described by the Brinkman equations ${ }^{23}$ for flow in a packed-bed:

$$
\begin{gathered}
\frac{\rho}{\varepsilon_{p}}\left[\frac{\partial \underline{\mathbf{u}}}{\partial t}+(\underline{\mathbf{u}} \cdot \nabla) \frac{\underline{\mathbf{u}}}{\varepsilon_{p}}\right] \\
=\nabla \cdot\left\{-p \underline{\mathbf{I}}+\mu\left[\nabla \underline{\mathbf{u}}+(\nabla \underline{\mathbf{u}})^{\tau}\right]-\frac{2}{3} \frac{\mu}{\varepsilon_{p}}(\nabla \cdot \underline{\mathbf{u}}) \underline{\mathbf{I}}\right\} \\
-\left(\frac{\mu}{\kappa_{b r}}+\frac{Q_{b r}}{\varepsilon_{p}^{2}}\right) \underline{\mathbf{u}} \\
\frac{\partial\left(\varepsilon_{p} \rho\right)}{\partial t}+\nabla \cdot(\rho \underline{\mathbf{u}})=Q_{b r}
\end{gathered}
$$

where $\kappa_{b r}$ is the permeability of porous media and $Q_{b r}$ is the volumetric mass source for the gas phase. Obviously, $Q_{b r}$ equals to the sum of volumetric mass sources for all individual species in the gas phase:

$$
Q_{b r}=\sum_{k} R_{k} \quad(k=1,2)
$$

The electrochemical sub-model at the RSOFC boundary.-The mass flux of the gas phase species at the RSOFC boundary are coupled to the electrochemical current density, $i_{n}$ :

$$
\begin{gathered}
-\underline{\mathbf{n}} \cdot \underline{\mathbf{N}}_{1}=-\frac{i_{n} M_{1}}{2 F} \\
-\underline{\mathbf{n}} \cdot \underline{\mathbf{N}}_{2}=\frac{i_{n} M_{2}}{2 F}
\end{gathered}
$$

where $\underline{\mathbf{n}}$ is the unit normal vector pointing out of the RSOFC boundary, $i_{n}$ is the anode current density of the RSOFC, and $F$ is the Faraday's constant. We assume that the mass transfer of $\mathrm{O}^{2-}$ ions from the cathode to the anode of the RSOFC during discharge is sufficiently fast due to the small thickness of the RSOFC, so the concentration of $O^{2-}$ ions in the RSOFC can be regarded as a constant. The anode current density of anode, $i_{n}$, is governed by the Butler-Volmer equation: ${ }^{24}$

$$
\begin{aligned}
i_{n}= & i_{n, 0}\left\{\exp \left[\frac{2 \alpha_{a} F}{R T}\left(\phi_{n}-E_{\mathrm{eq}, n}\right)\right]\right. \\
& \left.-\exp \left[-\frac{2\left(1-\alpha_{a}\right) F}{R T}\left(\phi_{n}-E_{\mathrm{eq}, n}\right)\right]\right\}
\end{aligned}
$$

where $\phi_{n}$ is the solid phase electric potential of the anode, $E_{\mathrm{eq}, n}$ is the equilibrium potential of the anode, $i_{n, 0}$ is the exchange current density for the anode, and $\alpha_{a}$ is the anodic transfer coefficient for the anode. As the concentration of $O^{2-}$ ions is a constant, the exchange current density of the anode depends only on the composition of the gas phase species through the following expression:

$$
i_{n, 0}=i_{n, 0}^{\text {ref }} x_{1}^{1-\alpha_{a}} x_{2}^{\alpha_{a}}
$$

where $i_{n, 0}^{\text {ref }}$ is the reference-state (where $x_{1}=1$ and $\alpha_{a}=0$ ) exchange current density. The equilibrium potential of the anode follows the Nernst equation:

$$
E_{\mathrm{eq}, n}=E_{\mathrm{eq}, n}^{\theta}+\frac{R T}{2 F} \ln \left(x_{2} / x_{1}\right)
$$

where $E_{\mathrm{eq}, n}^{\theta}$ is the equilibrium potential at the standard state. The solid phase materials of electrodes usually have large electrical conductivities, so the solid phase electric potential of anode, $\phi_{n}$, can be regarded as a function that depends on time only and can be solved by the limiting electrical equation:

$$
\frac{1}{A_{\partial \Omega_{E}}} \iint_{\partial \Omega_{E}} i_{n} d S=-i_{\text {app }}
$$

where $i_{\text {app }}$ is the applied current density on the RSOFC, $\partial \Omega_{E}$ is the symbol of the RSOFC boundary, and $A_{\partial \Omega_{E}}$ is the area of the RSOFC boundary. In this paper, $i_{\text {app }}$ is defined as positive for charging and negative for discharging.

Similar to the anode, the cathode current density is also governed by the Butler-Volmer equation:

$$
\begin{aligned}
i_{p}= & i_{p, 0}\left\{\exp \left[\frac{2 \beta_{a} F}{R T}\left(\phi_{p}-E_{\mathrm{eq}, p}\right)\right]\right. \\
& \left.-\exp \left[-\frac{2\left(1-\beta_{a}\right) F}{R T}\left(\phi_{p}-E_{\mathrm{eq}, p}\right)\right]\right\}
\end{aligned}
$$

where $\beta_{a}$ is the anodic transfer coefficient for the cathode, and other symbols have the same physical meanings as in equation 28 . The limiting electrical condition for the cathode is:

$$
\frac{1}{A_{\partial \Omega_{E}}} \iint_{\partial \Omega_{E}} i_{p} d S=i_{\text {app }}
$$

In this work, the cathode of the RSOFC works under constant oxygen partial pressure; therefore the exchange current density $\left(i_{p, 0}\right)$ and equilibrium potential $\left(E_{\mathrm{eq}, p}\right)$ of the cathode are both constants. When a constant current density is applied to the battery, the value of $\phi_{p}$ is obtained as a constant from eqs. 32 and 33. The voltage of the battery, $E$, is defined as the difference of solid phase electrical potentials between the cathode and the anode of the RSOFC:

$$
E=\phi_{p}-\phi_{n}
$$

while the full-cell Nernst potential, $U$, is expressed as:

$$
U=E_{\mathrm{eq}, p}-E_{\mathrm{eq}, n}
$$

and the overvoltage of the battery, $\eta$, is defined as

$$
\eta=E-U
$$

The gas circulation.-According to the assumption made previously, the "T"-tube is simplified to a CST where the pressure is constant and gas phase composition is uniform; therefore, the mass fraction gradients are zero at the boundary of the gas outlet and the absolute pressure at the gas outlet boundary is set at $p_{\text {ref }}$. The mass balance for a CST is described by the following lumped model: ${ }^{19}$

$$
\frac{d \bar{c}_{i}}{d t}=\frac{1}{\tau}\left(c_{i, f}-\bar{c}_{i}\right) \quad(i=1,2)
$$

where $\bar{c}_{i}$ is the mean molar concentration of species $i$ in the CST, $c_{i, f}$ is the mean molar concentration of species $i$ at the gas outlet boundary, and $\tau$ is the mean residence time of the CST. In this model, $\tau$ is expressed as:

$$
\tau=\frac{V_{R}}{Q_{f}}
$$

where $V_{R}$ is the volume of the CST, and $Q_{f}$ is the volumetric flow rate of the gas phase at the gas outlet boundary. The mass fractions of species in the circulated gas flow are same as those in the CST; therefore, the inflow condition at the gas inlet boundary of the venting pipe is set as:

$$
\left.\omega_{i}\right|_{\partial \Omega_{i n}}=\bar{c}_{i} \frac{M_{i}}{M_{n}}\left(\frac{R T}{p_{A}}\right)
$$

where $\partial \Omega_{\text {in }}$ is the symbol for the gas inlet boundary. The normal inflow velocity at the gas inlet boundary, $U_{0}$, is set as a constant.

Model inputs._-Values for the parameters involved in eq. 4 through 39 are listed in Table. The value for the binary diffusivity, $D_{1,2}$, is obtained from ref. 25 According to ref. 5, the full-cell Nernst potential, $U$, is measured as $0.970[\mathrm{~V}]$ at the mole fraction ratio $x_{1} / x_{2}=0.65 / 0.35$, therefore, it can be calculated that $E_{\mathrm{eq}, n}^{\theta}=-0.941[\mathrm{~V}]$ vs $E_{\mathrm{eq}, p}$. In this work, $E_{\mathrm{eq}, p}$ is set to 0 as the reference electric potential. 
Table I. Parameters used for the simulations.

\begin{tabular}{ccc} 
Parameter & Value & Unit \\
\hline$D_{1,2}$ & $7.6 \times 10^{-4}$ & $\mathrm{~m}^{2} / \mathrm{s}$ \\
$E_{\mathrm{eq}, n}^{\theta}$ & -0.941 & $\mathrm{~V}$ \\
$E_{\mathrm{eq}, p}$ & 0 & $\mathrm{~V}$ \\
$F$ & 96487 & $\mathrm{C} / \mathrm{mol}$ \\
$i_{p, 0}$ & 78.96 & $\mathrm{~A} / \mathrm{m}^{2}$ \\
$i_{n, 0}^{\text {ref }}$ & $4.623 \times 10^{3}$ & $\mathrm{~A} / \mathrm{m}^{2}$ \\
$k$ & 10 & $1 / \mathrm{s}$ \\
$k^{-1}$ & 5.38 & $1 / \mathrm{s}$ \\
$p_{\text {ref }}$ & 1.0 & $\mathrm{~atm}$ \\
$R$ & 8.3143 & $\mathrm{~J} / \mathrm{mol} / \mathrm{K}$ \\
$T$ & 800 & $\mathrm{o}$ \\
$V_{R}$ & $5.63 \times 10^{-6}$ & $\mathrm{~m}$ \\
$\alpha_{a}$ & 0.5 & \\
$\beta_{a}$ & 0.5 & $\mathrm{~m}$ \\
$\kappa_{b r}$ & $1.18 \times 10^{-11}$ & $\mathrm{~Pa} \cdot \mathrm{s}$ \\
$\mu$ & $1.0 \times 10^{-4}$ & $\mathrm{~kg} / \mathrm{m}^{3}$ \\
$\rho_{F e}$ & $7.8 \times 10^{3}$ & $\mathrm{~kg} / \mathrm{m}^{3}$ \\
$\rho_{F e O}$ & $5.5 \times 10^{3}$ & \\
$\varepsilon_{p, 0}$ & 0.7 & \\
& &
\end{tabular}

\section{Results and Discussion}

The parameter values for this model are listed in Table I. A discharge processes at $i_{\text {app }}=-200 \mathrm{~mA} / \mathrm{cm}^{2}$ was simulated to compare with the experimental data. The RSOFC applied in the experiment is a commercially available NextCell Electrolyte Supported Button Cell (Fuel Cell Materials, Ohio, USA). Table II gives the composition and thickness of the functional layers in the RSOFC. Other numerical results from the simulation are used for the extended studies of this flow battery's electrical and chemical behavior. The RCU in the experimental battery has an initial Fe loading of $0.907 \mathrm{~g}$ and an initial porosity of 0.7 , and the operating temperature for the system is $800^{\circ} \mathrm{C}$. The end-of-discharge condition in our simulation is set at $E=0.24 \mathrm{~V}$ and the velocity of inflow, $U_{0}$, is set at $0.1 \mathrm{~m} / \mathrm{s}$. The initial mass fractions of hydrogen and steam are $\omega_{1}=0.999$ and $\omega_{2}=0.001$, respectively. Discharges at other current densities were also simulated as case studies.

The electrochemical results. - The simulated vs experimental voltage profiles under $i_{\text {app }}=-200 \mathrm{~mA} / \mathrm{cm}^{2}$ discharge are presented in Figure 4a. It is evident that the model produces results in good agreement with the measured data. The full discharge continues for about 4.5 hour to reach the stop condition. According to the plots, the battery voltage decreases almost linearly for the depth of discharge (DOD) ranging between 0 and $80 \%$, beyond which the voltage falls sharply as a result of the depletion of hydrogen at the anode of the RSOFC. The profiles for the surface-averaged full-cell Nernst potential (note that even the battery is not really under open-circuit, $U$ can still be calculated from Nernst equation) and overvoltage vs time are shown in Figure 4b. At the beginning of discharge, the full-cell Nernst potential, which is a logarithmic function of partial pressures of $\mathrm{H}_{2} \mathrm{O}$ and $\mathrm{H}_{2}$ according to the Nernst equation, drops very quickly because the sensitivity $\partial U / \partial x_{2}$ is infinitely large in magnitude when the mole fraction of steam, $x_{2}$, is close to zero. For a similar reason, the overvoltage of the battery, which is an inverse hyperbolic function of applied current density according to the Butler-Volmer equation, increases quite sharply as the discharge begins and counterbalances

\begin{tabular}{lcc}
$\begin{array}{l}\text { Table II. Compositions and dimensions of the commercial } \\
\text { NextCell. }\end{array}$ & \\
& Composition & Thickness $(\mu \mathrm{m})$ \\
Component & Ni-YSZ/Ni-GDC (interlayer) & 50 \\
\hline Fuel electrode & Hionic $\mathrm{ZrO}_{2}$-based & $150(+/-15)$ \\
Electrolyte & LSM/LSM-GDC(interlayer) & 50 \\
Air electrode &
\end{tabular}

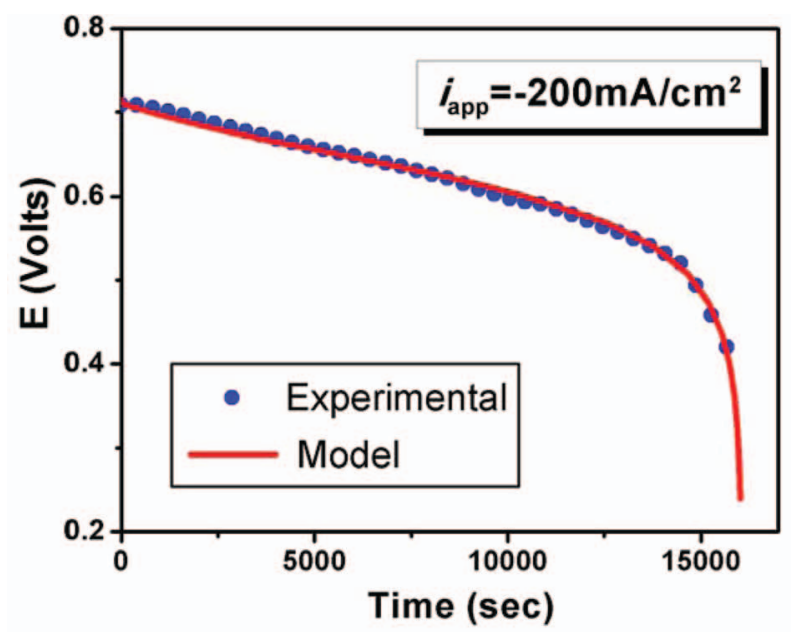

(a)

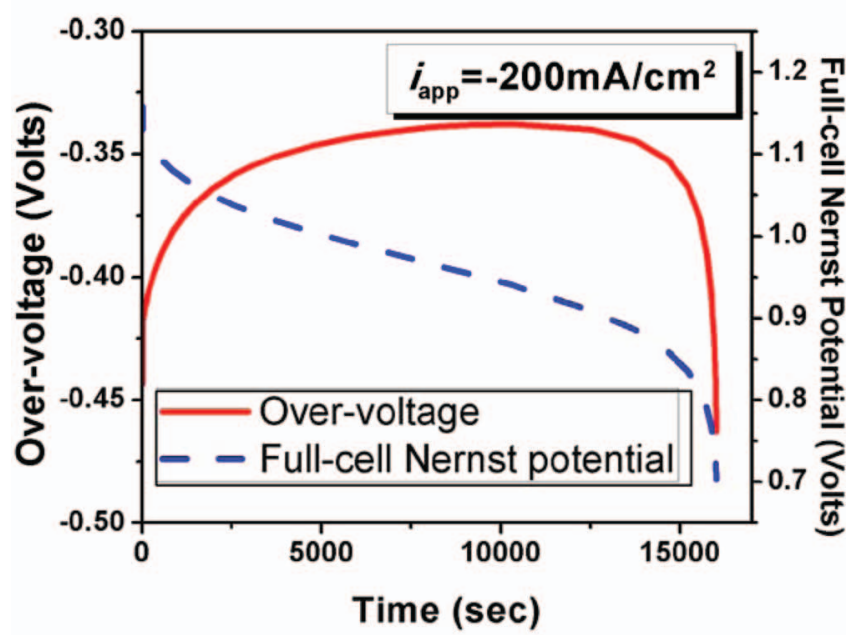

(b)

Figure 4. Results from the electrochemical sub-model: (a) The simulated vs experimental voltage of RSOFC operated at $i_{\text {app }}=-200 \mathrm{~mA} / \mathrm{cm} 2$, (b) The simulated surface-average over voltage and full-cell Nernst potential of RSOFC.

the drop in the full-cell Nernst potential. As a result, the voltage of battery only changes slightly at the beginning of the discharge. According to Figure $4 \mathrm{~b}$, the calculated internal resistance of this battery at $i_{\text {app }}=-200 \mathrm{~mA} / \mathrm{cm}^{2}$ is about $1.8 \Omega \cdot \mathrm{cm}^{2}$. It is noted that from the beginning to the end of this discharge, the voltage of the battery drops by $0.48 \mathrm{~V}$ while the overvoltage of the battery has a magnitude of $0.35 \mathrm{~V}$. Obviously, the discharge profiles will change significantly at different applied current densities. The end-of-discharge distributions of the full-cell Nernst potential and the electrochemical current density on the surface of the RSOFC anode are presented in Figure 5; the non-uniformities of these electrochemical variables are caused by the distributions of the gas-phase species concentrations on the electrode surface.

The CFD results. - The end-of-discharge distribution of the fluid velocity fields throughout the tubular reactor and the upper part of the returning tube is presented in Figure 6a. The velocity magnitude changes significantly when the gas flow leaves the narrow returning tube and enters the large space of the tubular reactor. The direction of the gas flow in the top regions of the tubular reactor are shown by the 2D streamline plots in Figure 6b; the main stream of the gas flow travels through the space between the RCU and the tube wall while only a small part of the gas phase enters the RCU. The distribution of the modified pressure in the gas phase is shown in Figure 6c, the 


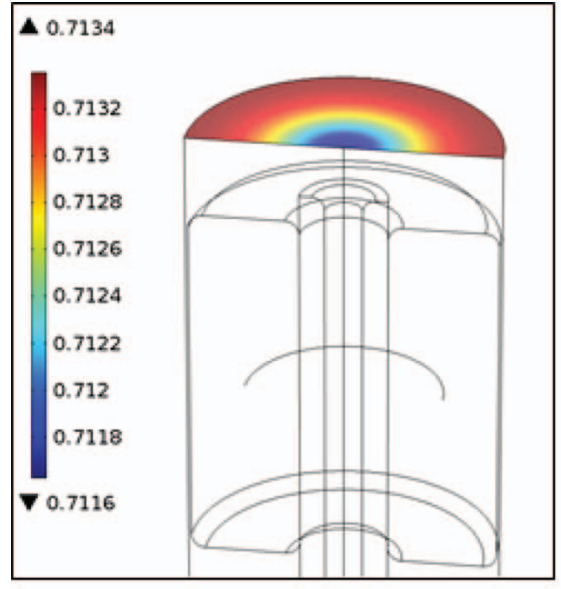

Full-cell Nernst potential ( $V$ )

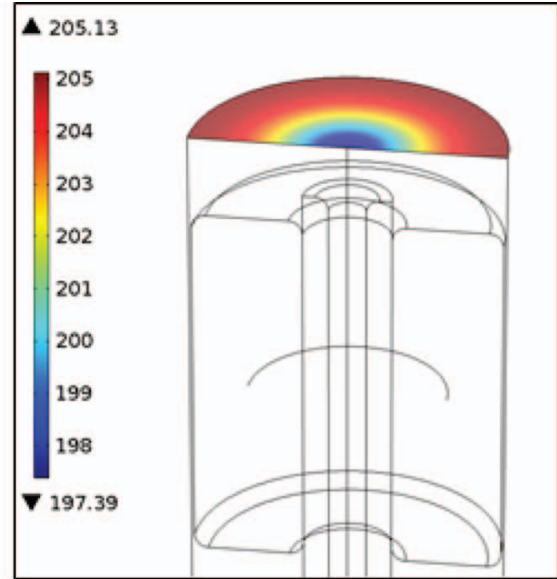

Current density $\left(\mathrm{mA} / \mathrm{cm}^{2}\right)$

Figure 5. End-of-discharge distribution of full-cell Nernst potential and electrochemical current density.

pressure drops significantly through the RCU domain because of the resistive forces in the porous phases.

The mass transfer results. - The end-of-discharge distributions of the hydrogen and steam mole fractions are presented in Figure $7 \mathrm{a}$ and $7 \mathrm{~b}$, and the profile for distributed Fe-to-FeO conversion in the RCU is presented in Figure 7c. As shown in these plots, both the hydrogen and the Fe have about $98 \%$ conversions at the end of discharge. As steam is generated through the electrochemical reaction at the RSOFC boundary, the mole fraction of steam at the top of the tubular reactor

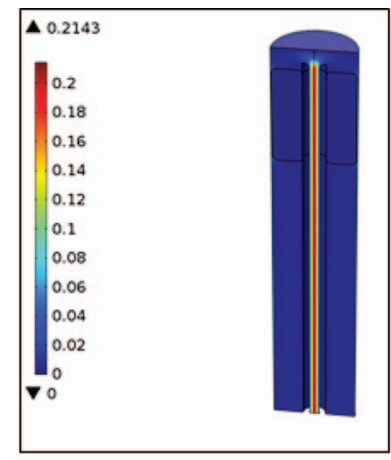

(a) Magnitude of velocity $(\mathrm{m} / \mathrm{s})$

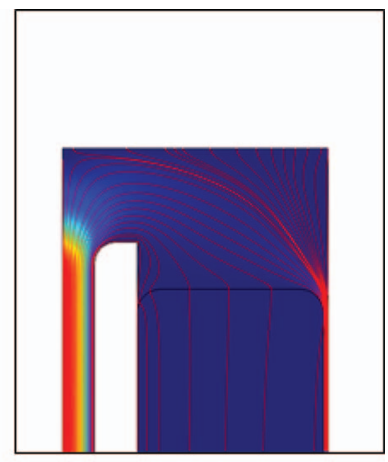

(b) 2D streamline plot for velocity field is larger than that at the bottom by about 0.025 ; and the molar fraction of hydrogen has an opposite distribution profile. In accordance with the distributions of the gas-phase species, the $\mathrm{Fe}-$ to- $\mathrm{FeO}$ conversion at the top of the RCU is slightly higher than that at the bottom because a larger steam/hydrogen ratio drives the reaction 3 to the right hand.

The overall generation rate of hydrogen in the RCU and the overall consumption rate of hydrogen at the RSOFC anode are compared in Figure 8a. As shown in this plot, the generation rate of hydrogen is comparatively small at the beginning of discharge due to the low concentration of steam, but increases very quickly to catch the consumption rate of hydrogen. The overall material balance in the gas phase is close to a pseudo-steady state (PSS) where the generation and consumption rates are equal for both hydrogen and steam.

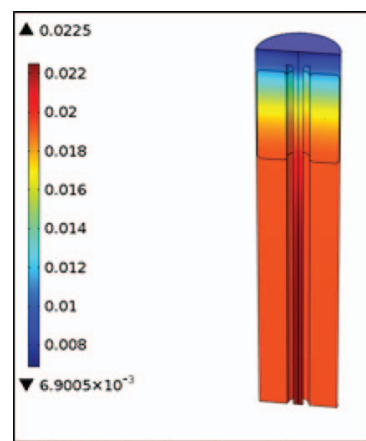

(a) Mole fraction of $\mathrm{H}_{2}$

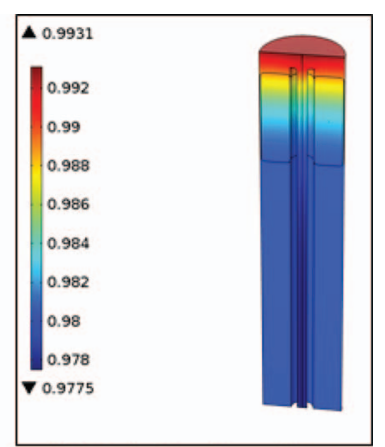

(b) Mole fraction of $\mathrm{H}_{2} \mathrm{O}$

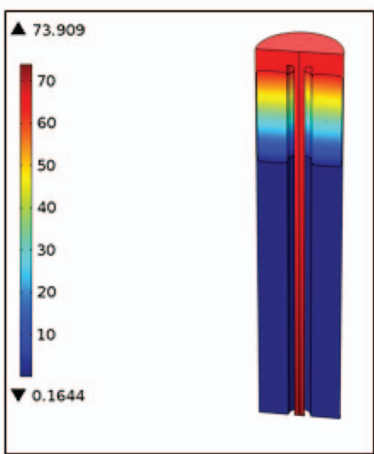

(c) Modified pressure $(\mathrm{Pa})$

Figure 6. The end-of-discharge distributions of the velocity and pressure of the gas flow.

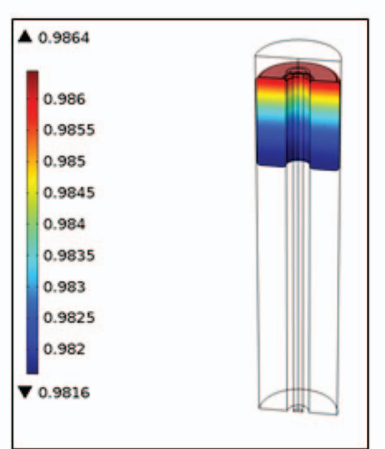

(c) Conversion of Fe

Figure 7. End-of-discharge distributions of (a) molar fraction of hydrogen, (b) molar fraction of steam, and (c) conversion rate of Fe. 


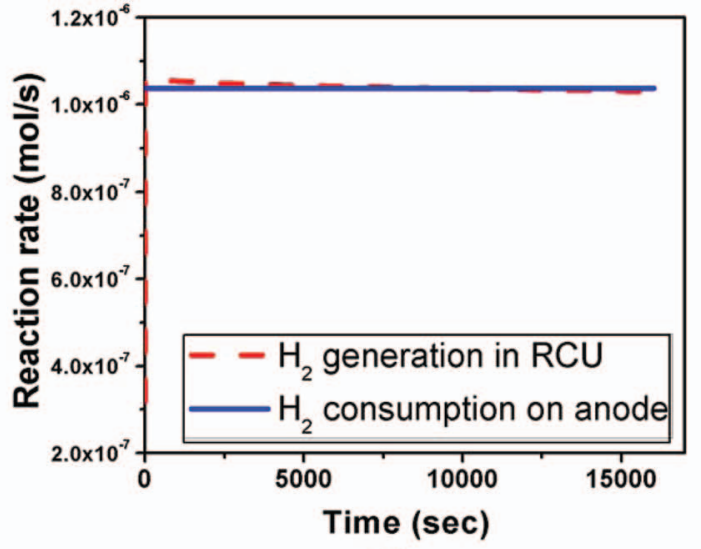

(a)

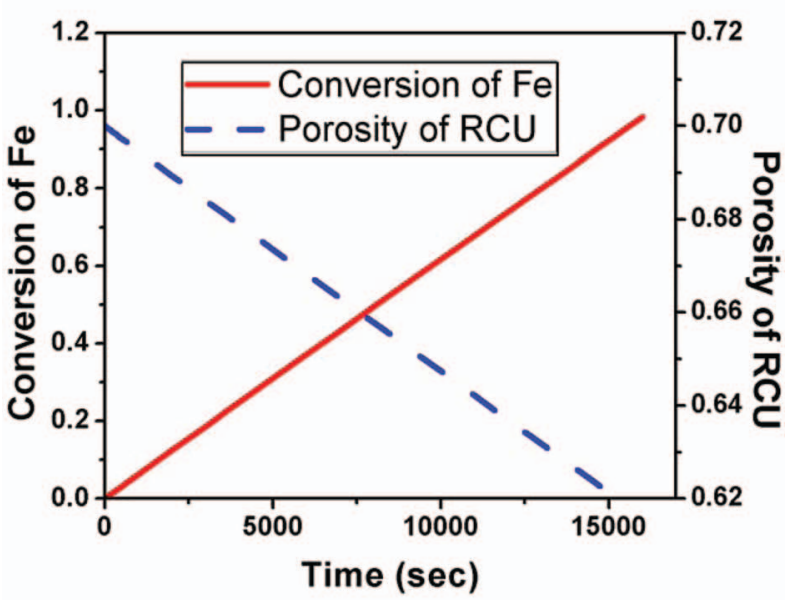

(b)

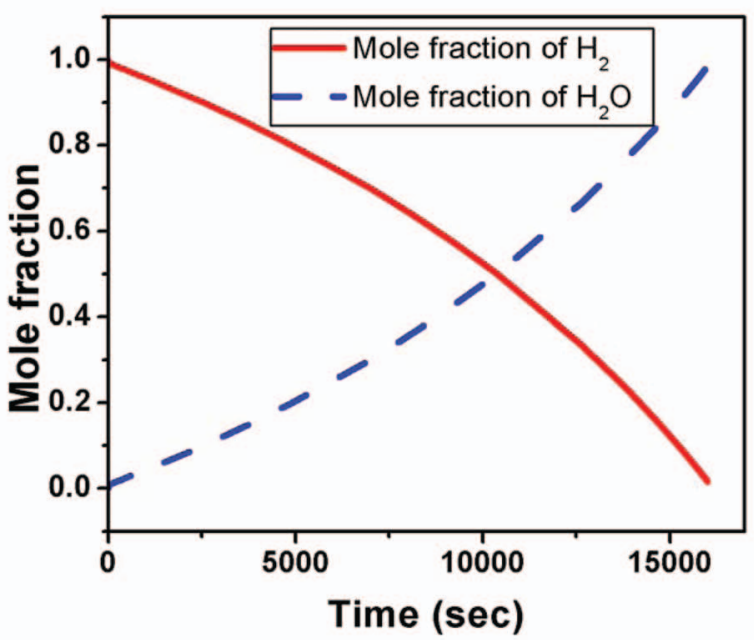

(c)

Figure 8. Mass transfer behavior of the battery (a) overall generation/ consumption rates of hydrogen, (b) volume-average molar fractions of hydrogen and steam in the RCU, (c) volume-average Fe conversion and porosity in the RCU.

As the hydrogen-generating RCU is located close to the hydrogenconsuming RSOFC and the gas phase diffusivity at high temperature is large $\left(7.6 \times 10^{-4} \mathrm{~m}^{2} / \mathrm{s}\right)$, the mass transfer between the RCU and the RSOFC is sufficiently fast and the overall mass balance of the system is limited by the reactions except for the initial stage in which the concentration gradients in gas phase are not fully developed; therefore,

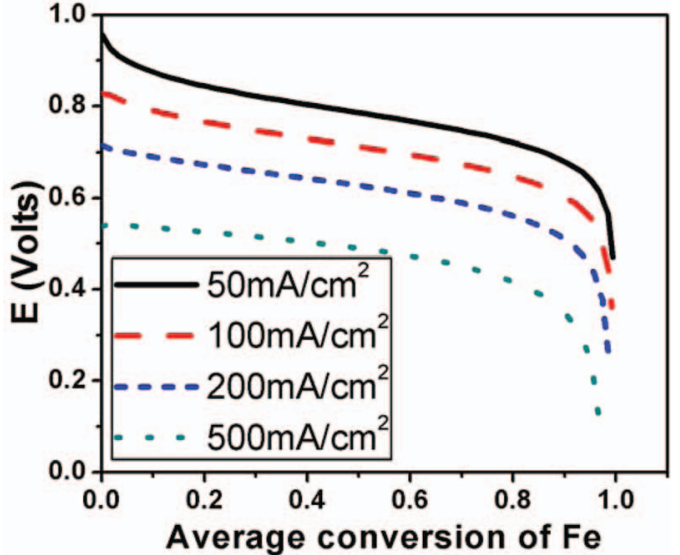

(a)

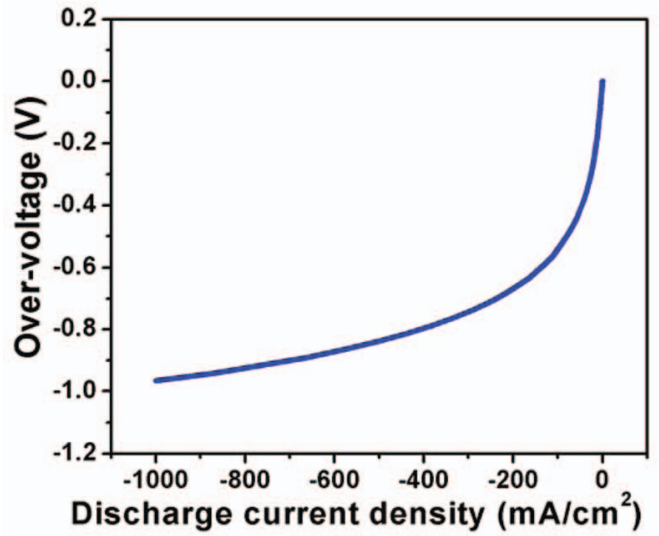

(b)

Figure 9. Discharge profiles of the battery at different applied current densities (a) and the dependency of initial over-voltage on applied discharge current density (b).

after short time delay, the gas phase stays in the PSS until the end of discharge. As a result of the PSS in the gas phase, the volume-average Fe conversion and porosity in the RCU change linearly with time (see Figure 8b). However, as shown in Figure 8c, the average molar fractions of gaseous species in the RCU change with time in nonlinear profiles. According to eq. 16, during the PSS, the reaction rate $r_{1}$ is close to a constant, while $\xi_{F e}$ is a linear function of time and the total molar concentration of gas mixture, $c_{1}+c_{2}$, is close to a constant due to the equal-molar conversion between hydrogen and steam; it can be easily derived that $c_{1}$ and $c_{2}$ change with time through the hyperbolic correlations.

The discharge profiles at different current densities. - Discharge profiles of this battery at $i_{a p p}=-50,-100,-200$, and $-500 \mathrm{~mA} / \mathrm{cm}^{2}$ are compared in Figure 9; in these simulation results, the battery was fully discharged (program stops when the mole fraction of hydrogen at the RSOFC boundary drops to 0.001 ). The plateaus of the discharge curves drop by $0.30 \mathrm{~V}$ as the applied current density changes from -50 to $-500 \mathrm{~mA} / \mathrm{cm}^{2}$, which is consistent with the analysis given in the section for the electrochemical results. As the theoretical capacity of the battery is limited only by the loading of the Fe material, the change of applied current does not cause big differences in the fulldischarge capacity. However, in practical operations, the batteries are often set under voltage control; therefore, the discharge capacities may shift significantly with the input current.

\section{Conclusion}

In this work, a rigorous multi-physics model for a solid oxide redox flow battery system was developed based on the fundamental 
theories of reaction engineering. The major functioning part of the battery is treated as a tubular packed-bed reactor combined with a working planar disk electrochemical cell. The gas circulation system of the battery is simplified to a continuous-stirred tank described by lumped material balance sub-models. The developed model shows is suitable for studying electrical and chemical behavior of this new flow battery system, and can be extended to other battery configurations. The simulated voltage profile under a $200 \mathrm{~mA} / \mathrm{cm}^{2}$ discharge agrees well with the experimental data.

\section{List of Symbols}

\section{Symbol \\ Description}

$A_{\partial \Omega_{E}}$

$c_{i}$

$\bar{c}_{i}$

$c_{i, f}$

$c_{F e}$

$\underline{\mathbf{d}}_{k}$

$\underline{\mathbf{d}}_{k}$

$\tilde{D}_{i, k}$

$\tilde{D}_{i, k}^{e f f}$

\section{E}

$E_{\text {eq, } n}$

$E_{\mathrm{eq}, p}$

$E_{\text {eq }, n}^{\theta}$

$F$

$i_{\text {app }}$

$i_{n} i_{p}$

$i_{n, 0} i_{p, 0}$

$i_{n, 0}^{\text {ref }}$

I

$\overline{\mathbf{j}}_{i}$

$k^{-1}$

$M_{F e}$

$\mathrm{M}_{\mathrm{FeO}}$

$M_{k}$

$M_{n}$

n

$n_{F e}$

$n_{\mathrm{FeO}}$

$\underline{\mathbf{N}}_{i}$

$p$

$p_{A}$

$p_{\text {ref }}$

$Q_{b r}$

$Q_{f}$

$r_{i}$

$R$

$R_{i}$

the CST

the gas outlet boundary

bulk volume of RCU

diffusivities

Voltage of the battery

for anode

Faraday's constant

anode/cathode of RSOFC

anod/cathode

Identity matrix reaction

regenerative reaction

Molar mass of $\mathrm{Fe}$

Molar mass of $\mathrm{FeO}$

RSOFC boundary

Amount of $\mathrm{Fe}$

Amount of $\mathrm{FeO}$

Total mass flux of species $i$

Modified pressure

Absolute pressure

Reference pressure gas outlet boundary

Universal gas constant
Area of the RSOFC boundary

Molar concentration of gas phase species $i \mathrm{~mol} / \mathrm{m}^{3}$

Mean molar concentration of species $i$ in

Mean molar concentration of species $i$ at

Total amount of $\mathrm{Fe}$ and $\mathrm{FeO}$ per unit

Diffusive driving force acting on species $k$

Maxwell-Stefan diffusivities

Multi-component Fick diffusivities

Effective multi-component Fick

Equilibrium potential of the anode/cathode

Standard-state equilibrium potential

Applied current density on the battery

Electrochemical current densities for the

Exchange current density for the

Reference-state exchange current density

Diffusive mass flux vector for species $i \quad \mathrm{~kg} / \mathrm{m}^{2} / \mathrm{s}$

Rate constant for the forward regenerative

Rate constant for the reverse

Molar mass for gas phase species $k$

Mean molar mass of gas mixture

Unit normal vector pointing out of the

Volumetric mass source for the gas phase

Volumetric flow rate of the gas phase at the

Volumetric molar reaction rate of species $i \mathrm{~mol} / \mathrm{m}^{3} / \mathrm{s}$

Volumetric mass source of species $i$

V
$\mathrm{V}$

$\mathrm{A} / \mathrm{m}^{2}$

$\mathrm{A} / \mathrm{m}^{2}$

$\mathrm{A} / \mathrm{m}^{2}$

$\mathrm{A} / \mathrm{m}^{2}$

$1 / \mathrm{s}$

$1 / \mathrm{s}$

$\mathrm{kg} / \mathrm{mol}$

$\mathrm{kg} / \mathrm{mol}$

$\mathrm{kg} / \mathrm{mol}$

$\mathrm{kg} / \mathrm{mol}$

mol

mol

$\mathrm{kg} / \mathrm{m}^{2} / \mathrm{s}$

$\mathrm{Pa}$

$\mathrm{Pa}$

$\mathrm{Pa}$

$\mathrm{kg} / \mathrm{m}^{3} / \mathrm{s}$

$\mathrm{m}^{3} / \mathrm{s}$

$\mathrm{J} / \mathrm{mol} / \mathrm{K}$

$\mathrm{kg} / \mathrm{m}^{3} / \mathrm{s}$
$T$

$\underline{\mathbf{u}}$

$\bar{U}$

$U_{0}$

$V_{R}$

$V_{\mathrm{RCU}}$

$x_{k}$

$\alpha_{a}$

$\beta_{a}$

$\varepsilon_{p}$

$\varepsilon_{p, 0}$

$\phi_{n} \phi_{p}$

$\eta$

$\kappa_{b r}$

$\mu$

$\rho$

$\rho_{F e}$

$\rho_{\mathrm{FeO}}$

$\tau$

$\omega_{i}$

$\xi_{F e}$

$\xi_{F e, 0}$

$\partial \Omega_{\mathrm{E}}$

$\partial \Omega_{\text {in }}$

$\nabla$

Subscript

$i$

k
Time

Temperature

Velocity vector of the fluid flow,

Full-cell Nernst potential of the battery

Normal inflow velocity at the gas

inlet boundary

Volume of the CST

Bulk volume of RCU

Mole fraction of species $k$

Anodic transfer coefficient for the anode

Anodic transfer coefficient for the cathode

Porosity of the RCU

Initial porosity of the RCU

Solid phase electric potential of the

anode/cathode

Over voltage of the battery

Permeability of porous media

Viscosity of gas phase

Density of domain gasphase

Density of Fe

Density of $\mathrm{FeO}$

Mean residence time of CST

Mass fraction of species $i$

Conversion for $\mathrm{Fe}$ to $\mathrm{FeO}$

Initial conversion for $\mathrm{Fe}$ to $\mathrm{FeO}$

Symbol for the RSOFC boundary

Symbol for the gas inlet boundary

The gradient operator

$1 / \mathrm{m}$

$\mathrm{s}$
$\mathrm{K}$
$\mathrm{m} / \mathrm{s}$
$\mathrm{V}$
$\mathrm{m} / \mathrm{s}$
$\mathrm{m}^{3}$
$\mathrm{~m}^{3}$

$\mathrm{S}$

$\mathrm{m} / \mathrm{s}$

$\mathrm{m} / \mathrm{s}$

$\mathrm{m}^{3}$

\section{Description}

Index for gaseous species $(i=1$ for hydrogen and $i=2$ for steam)

Dummy index for species

\section{References}

1. Z. Peng, S. A. Freunberger, Y. Chen, and P. G. Bruce, Science., 337, 563 (2012).

2. J.-S. Lee, S. Tai Kim, R. Cao, N.-S. Choi, M. Liu, K. T. Lee, and J. Cho, Advanced Energy Materials, 1, 24 (2011).

3. F. Cheng and J. Chen, Chemical Society Reviews., 41, 2172 (2012).

4. Y. C. Lu, H. A. Gasteiger, and Y. Shao-Horn, J. Am. Chem. Soc., 133, 19048 (2011).

5. N. Xu, X. Li, X. Zhao, J. B. Goodenough, and K. Huang, Ener. \& Environ. Sci., 4 4942 (2011)

6. X. Zhao, N. Xu, X. Li, Y. Gong, and K. Huang, RSC Adv., 2, 10163 (2012).

7. X. Zhao, Y. Gong, X. Li, N. Xu, and K. Huang, J. Electrochem. Soc., 160, A1241 (2013).

8. X. Zhao, N. Xu, X. Li, Y. Gong, and K. Huang, ECS. Trans., 50, 115 (2013).

9. X. Zhao, N. Xu, X. Li, Y. Gong, and K. Huang, ECS. Trans., 45, 113 (2013).

10. X. Zhao, X. Li, Y. Gong, N. Xu, K. Romito, and K. Huang, Chem. Commun., 49, 5357 (2013).

11. H. Landes and R. Reichenbacher, ECS. Trans., 50, 47 (2013).

12. A. Inoishi, Y. W. Ju, S. Ida, and T. Ishihara, J. Power Sources., 229, 12 (2013).

13. A. Inoishi, S. Ida, S. Uratani, T. Okano, and T. Ishihara, Phys. Chem. Chem. Phys., 14, 12818 (2012).

14. A. Inoishi, Y. W. Ju, S. Ida, and T. Ishihara, Chem. Commun., 49, 4691 (2013).

15. A. Inoishi, S. Ida, S. Uratani, T. Okano, and T. Ishihara, RSC Adv., 3, 3024 (2013).

16. W. Drenckhahn, H. Greiner, M. Kuhne, H. Landes, A. Leonide, K. Litzinger, C. Lu, C. Schuh, J. Shull, and T. Soller, ECS. Trans., 50, 125 (2013).

17. A. Inoishi, Y. Okamoto, Y. W. Ju, S. Ida, and T. Ishihara, RSC Adv., 3, 8820 (2013).

18. H. Ohmori, S. Uratani, and H. Iwai, J. Power Sources., 208, 383 (2012).

19. J. B. Rawlings and J .G. Ekerdt, Chemical reactor analysis and design fundamentals., Nob Hill Pub., (2002).

20. Chemical Reaction Engineering Module User's Guide (for COMSOL 4.3b)., 202-209 (2013).

21. C. F. Curtiss and R. B. Bird, Ind. Chem. Res., 38, 2515 (1999).

22. F. M. White, Viscous Fluid Flow (3rd edition)., Tata McGraw-Hill Education, 1974.

23. M. L. Bars and M. Worster, Journal of Fluid Mechanics., 550, 149 (2006).

24. P. Geoffrey. Electrochemical engineering principles (Vol. 1)., Englewood Cliffs, NJ: Prentice Hall, (1991).

25. W. He, K.-J. Yoon, R. S. Eriksen, S. Gopalan, S. N. Basu, and U. B. Pal, J. Power Sources., 195, 532 (2010). 